\title{
OBSERVATIONAL DATA IMPLYING THE NGC 1275 NUCLEUS COMPLEXITY
}

\author{
I.PRONIK \\ Crimean Astrophysical Observatory \\ 334413, p/o Nauchny, Crimea, Ukraine
}

Introduction. The central unresolved source of the galaxy NGC 1275 in red has a dimension of about $8^{\prime \prime}$, but in blue its bright part is about of $0 . " 5$. HST observations, obtained with the filter $\lambda 2200 \AA$ cannot reveal the structure of the source inside $0 . " 26$. Now we have collected the observed features of NGC 1275 nucleus in optics and radio domains, which are possibly associated with the complex structure of the nucleus.

Two kinematical centers in the NGC 1275 nucleus. NGC1275 galaxy itself in $\mathrm{H}_{\alpha}$ light looks like Crab nebulae consisting of gaseous filaments elongated mainly in radial directions. Radial velocities of these filaments are proportional to its angular distances and have two kinematical centers located inside the $1^{\prime \prime}$ of the nucleus. Their radial velocities equal to +5255 and $+5110 \mathrm{~km} / \mathrm{s}$. The difference is $(140 \pm 20) \mathrm{km} / \mathrm{s}$.

Profiles of emission lines. The emission line profiles in spectra of NGC 1275 nucleus [OIII] $\lambda \lambda 4959$ and $5007 \AA \AA$ and $\mathrm{H}_{\alpha}$ have blue asymmetry. Dibaj, Esipov (1968) and V.Pronik (1987) showed that this asymmetry is caused by the components, radial velocities of which correspond to $4400 \mathrm{~km} / \mathrm{s},+5110 \mathrm{~km} / \mathrm{s}$ and $+5255 \mathrm{~km} / \mathrm{s}$. Two latter are the same as radial velocities of kinematical centers of gaseous filaments (see above).

Direct images of the NGC 1275 nucleus. Direct images obtained with the 6-m telescope through broad band filters $\left(\lambda_{e f}=4800 \AA\right.$ and $\lambda_{e f}$ $=6650 \AA$ ) show the elongation of the NGC 1275 nucleus, different in red and blue lights. Elongation in blue is seen at a Position Angle $(\mathrm{PA})=340^{\circ}$, elongation in red is seen at $\mathrm{PA}=220^{\circ}$.

Gaseous envelope of the NGC 1275 nucleus was investigated using unwidened spectra obtained in prime focus of the 6 - $\mathrm{m}$ telescope in 14 bright emission lines: $\mathrm{H}_{\alpha}, \mathrm{H}_{\beta},[\mathrm{OIII}] \lambda 5007 \AA$ and others. The dimension of the nucleus (FWHM) in $\mathrm{PA}=220^{\circ}$ was found to be near to that of equipment resolution, but in $\mathrm{PA}=340^{\circ}$ this dimension was essentially wider. FWHM 
for emission lines $\mathrm{H}_{\beta}$ and $\lambda 5007 \AA$ is $\sim 2^{\prime \prime}$ and for the line [OII] $3727 \AA-$ up to $3^{\prime \prime}$. One can conclude that there are emission line jets, or a possible bifurcation of the nucleus at $\mathrm{PA}=340^{\circ}$.

Spectral variability. The spectral data of NGC 1275 nucleus gathered for the period 1971-1982 showed, that there was essential variability of relative intensities of emission lines and ratios $\mathrm{I}_{\lambda} / \mathrm{I}_{\beta}$ depending on nucleus' brightness. It was obtained that $2 \leq \mathrm{I}_{[O I I I]} / \mathrm{I}_{\beta} \leq 7$. Lower values of ratio $\mathrm{I}_{[O I I I} / \mathrm{I}_{\beta}$ correspond to bright nucleus $\left(\mathrm{V}\left(5^{\prime \prime}\right) \leq 13 .{ }^{m} 5\right.$ - case 1$)$, and high ratios - to the weak nucleus $\left(\mathrm{V}\left(5^{\prime \prime}\right) \geq 13 .^{m} 5\right.$ - case 2$)$. In case 2 there is a pronounced correlation between $\mathrm{I}_{[O I I I]}$ and $\mathrm{I}_{\beta}$ - coefficient of correlation $\mathrm{r}$ $=0.76 \pm 0.08$. In case 1 coefficient of the correlation between $\mathrm{I}_{[O I I I]}$ and $\mathrm{I}_{\beta}$ is essentially less $-\mathrm{r}=0.42 \pm 0.16$.

Relative emission line intensities $I_{\lambda} / I_{\beta}$ show also differences in cases 1 and 2. If $\mathrm{V}\left(5^{\prime \prime}\right) \geq 13 .^{m} 5$, ratios $\mathrm{I}_{\lambda} / \mathrm{I}_{\beta}$ for emission lines of low excitation $3727+29$ [OII], $6300+64$ [OI], 6548+84 [NII] and $6717+31$ [SII] are higher than those ratios for bright nucleus, when $V\left(5^{\prime \prime}\right) \leq 13 .{ }^{m} 5$. The spectrum of the bright nucleus is like that of Sy 1 , but in the case of weak nucleus it is like that of Sy2. Considered data evidence the complex structure of the gaseous envelope or the nucleus itself.

The positions of the optical and radio nuclei of NGC 1275 . Many authors revealed a close resemblance in the complex bursts in $3.3 \mathrm{~mm}$ and in optical light curves of NGC 1275 nucleus, and the time shift between them is not more than 8 years. One can conclude that distance between radio and optical variable nuclei is not more than 8 light years, or $2.4 \mathrm{pc}$. Therefore, the projection of the distance between high variable optical and radio sources onto the sky is not more, than $0 . " 007$.

Shokin and Shulga (Dudinov et al., 1990) have obtained, that in 1989 the position of the optical nucleus of NGC 1275 was $0 . " 3 \mathrm{NW}$ from the position of radio VLBI nucleus. The error of the measurement was not more than 0."05. Taking into account, that the distance of variable optical nuc' . from radio one is not more than $0 . " 007$, one can suppose that NGC 127. galaxy has two optical peaks near the nucleus - one of high degree variable is located near the variable radio source and another - by $0 .{ }^{\prime \prime} 3 \mathrm{NW}$ from the first one.

Conclusions. All compiled material permit to suspect, that NGC 1275 nucleus is complex. An approximate sketch on relative positions of its principal details is presented.

\section{References}

Dibaj E.A., Esipov V.F., 1968. Astron.Tsirk., No 467.

Dudinov V.N. et al.,1990, Pi'sma Astron. Zh., 16, 114.

Pronik V.I., 1987. In Proc. IAU Sym 121, eds. E.Khachikian et al., 191. 